\title{
Experimental Measurements Of Wakefields In A Multimode, Dielectric Structure Driven By A Train Of Electron Bunches.
}

\author{
J.G. Power, M.E. Conde, W. Gai, A. Kanareyken†, R. Konecny, and P. \\ Schoessow
}

Argonne National Laboratory, 9700 S. Cass Ave. Argonne, IL 60439
† St. Petersburg Electrical Engineering University, 5 Prof. Popov St., St. Petersburg 197376, Russia

\begin{abstract}
We report on the experimental results of a new wakefield acceleration scheme. The multibunch driven, multimode, dielectric wakefield accelerator was demonstrated at the Argonne Wakefield Accelerator (AWA). In this experiment, a bunch train of 4,5 $\mathrm{nC}$ electron bunches, separated by $760 \mathrm{ps,} \mathrm{was} \mathrm{passed} \mathrm{through} \mathrm{a} 60 \mathrm{~cm}$ long dielectric-lined cylindrical waveguide. The separation was chosen to match the net acceleration wavelength of the multimode structure. By carefully measuring the energy spectrum of the 4 beams after they passed through the waveguide, we demonstrated that the wakefield is indeed enhanced by a train of periodically spaced electron bunches. The analysis of the multimode structure driven by a bunch train was done by a trivial extension to the existing theory since we are operating in the linear regime. This work represents the first experimental demonstration of this concept and also shows that multibunch operation of wakefield accelerators is worthy of further investigation.
\end{abstract}

\section{INTRODUCITON}

We report on a new collinear acceleration scheme [1,2] in which a multimode, dielectric structure is driven by a bunch train. - i.e. a multimode dielectric wakefield accelerator (multimode DWFA) driven by a bunch train. This scheme has two distinguishing features compared with conventional collinear wakefield devices: the use of closely spaced, multiple drive bunches and the intentional excitation of multiple cavity modes to create the net wakefield. That is not to say that higher order modes (HOM's) are not excited in all structure wakefield accelerators, that is a wellestablished fact taken into account by all modern analysis. Rather, it is that the HOM's in most structures are regarded as parasitic. The dimensions of the multimode DWFA structure are chosen so the excited cavity modes are nearly harmonic (i.e. equally spaced in frequency) and thus superimpose (linearly) periodically to produce sharply peaked fields (approximately equal to the electron pulse length). (In retrospect, a more appropriate name would have been the 'harmonic' DWFA since all structures have multiple modes, but the 'multimode' name has already been established.) The wakefield left behind the beam looks like an alternating series of delta functions rather than the sine-wave like wakefield of the single-mode device.

Submitted to the proceedings of the 9th Advanced Accelerator Concepts Workshop, Santa Fe, NM, June 10-16, 2000. 
This scheme has two particularly interesting characteristics: relaxed drive beam requirements and (possibly) improved $r f$ breakdown properties. The former characteristic comes about by dividing the single high charge bunch into a train of low charge bunches, thus making it easier to produce low emittance, short pulse bunches. (Note that the use of pulse trains can benefit both multimode and single mode accelerators such as the CLIC decelerator.) The later feature arises since the short duration of the individual wakefield pulses may slightly increase the $r f$ breakdown voltage. This is simply because the dielectric is exposed to the high field for a shorter time than in the single-mode case.

In the first section of this paper, we show how the analytic solution for the usual DWFA excited by a single drive bunch [3] can be extended to the calculation of multimode DWFA driven by a bunch train. In the remainder of the paper we describe multimode structure design, the experimental setup, and the experimental results compared to numerical simulations. We end with some concluding remarks about the merits of this scheme.

\section{MULTIBUNCH, MULTIMODE DEVICES}

In this section we show how to extend the analytic solution for the usual DWFA excited by a single drive bunch [3] to the calculation of a multimode DWFA driven by a bunch train.

\section{Direct Solution Of The Wave Equation}

One can determine the wakefield excitation of any cylindrically symmetric structure [3] by first finding the point charged particle solution (Green Function) of the wave equation in cylindrical coordinates. For a particle of charge $q$ located at position $z_{0}$ and moving with axial velocity $v$, the point charge density and current are written as, $\rho\left(r, z_{0}, t\right)=q \frac{\delta(r)}{r} \delta\left(z_{0}-v t\right)$ and $J_{z}=v \rho$ respectively. Defining $z=z_{0}-v t$, one can now solve the wave equation and write the Green function for the axial electric field as,

$$
G_{z}(r, z)=\sum_{n=1}^{\infty} G_{n}(r) \cos \left(k_{n} z\right)
$$

where $G_{n}(r)$ are the coefficients of the Fourier expansion and $k_{n}$ are the wave numbers of the $n^{\text {th }}$ mode, which are determined by the boundary conditions. Since all bunches travel along the cylinder axis, for the rest of the paper we need only consider fields at $r$ $=0$ and will drop the explicit dependence on $r$. Using (1), one can solve for the longitudinal wakefield, $W_{z}(z)$, for any arbitrary charge distribution $f(z)$ by taking the convolution of the Green function over the axial charge distribution, 


\section{DISCLAIMER}

This report was prepared as an account of work sponsored by an agency of the United States Government. Neither the United States Government nor any agency thereof, nor any of their employees, make any warranty, express or implied, or assumes any legal liability or responsibility for the accuracy, completeness, or usefulness of any information, apparatus, product, or process disclosed, or represents that its use would not infringe privately owned rights. Reference herein to any specific commercial product, process, or service by trade name, trademark, manufacturer, or otherwise does not necessarily constitute or imply its endorsement, recommendation, or favoring by the United States Government or any agency thereof. The views and opinions of authors expressed herein do not necessarily state or reflect those of the United States Government or any agency thereof. 


\section{DISCLAIMER}

Portions of this document may be illegible in electronic image products. Images are produced from the best available original document. 


$$
\begin{aligned}
& \text { (1) } 6 \text { Cof } \\
& \text { NOV } 0 \& 2000 \\
& W_{z}(z)=\int_{-\infty}^{z} d z^{\prime} f\left(z^{\prime}\right)\left\{\sum_{n=1}^{\infty} G_{n} \cos \left[k_{n}\left(z-z^{\prime}\right)\right]\right\}
\end{aligned}
$$

This is a general solution for the longitudinal wakefield of any DWFA since all modes are accounted for. The only difference between the multimode device and the single mode device is that in the former many modes $(n \sim 50)$ are excited while in the later only a few modes $(n \sim 3)$ are appreciably excited. It is trivial to extend this solution to a "bunch train" of $M$ bunches separated by a distance, $\lambda$, by writing the multibunch charge distribution as, $f(z)=\sum_{m=1}^{M} f_{m}(z-m \lambda)$. Finally, the wakefield excited by the bunch train at any point $z$ (which may be inside a bunch) is obtained by substituting this multibunch charge distribution into (2),

$$
\begin{gathered}
W_{z}(z)=\int_{-\infty}^{z} d z^{\prime} \sum_{m=1}^{M} f_{m}\left(z^{\prime}-m \lambda\right)\left\{\sum_{n=1}^{\infty} G_{n} \cos \left[k_{n}\left(z-z^{\prime}\right)\right]\right\} \\
\equiv \sum_{m=1}^{M} W_{z m}(z)
\end{gathered}
$$

where $W_{z m}$ is the longitudinal wakefield due to the $m^{\text {th }}$ bunch (located at $z=m \lambda$ ) acting alone. This form of writing makes our assumption of linear superposition explicit and we now see that absolutely no extension to the existing theory is required other than invoking linear superposition.

\section{DESIGN OF THE MULTIMODE STRUCTURE}

In this section we discuss how the multimode, dielectric-lined, cylindrical wakefield structure (Fig. 1) was designed using the analytic work derived in [3].

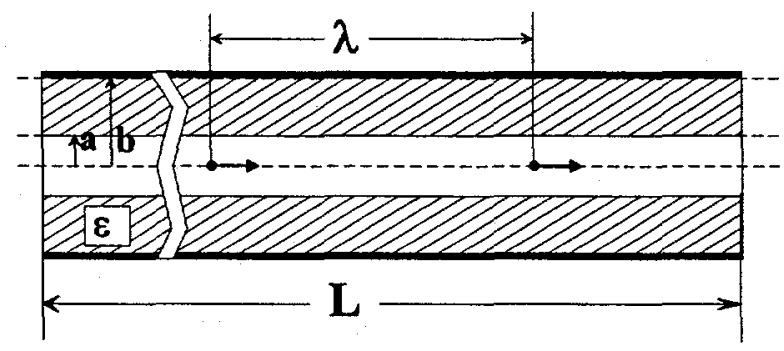

FIGURE 1. The Multimode Waveguide driven by a Bunch Train. The thick walled dielectric waveguide used in this experiment has inner radius $a=0.5 \mathrm{~cm}$, outer radius $b=1.44 \mathrm{~cm}$, length $L=60$ $\mathrm{cm}$ and dielectric constant $\varepsilon=38.1$. Two bunches from the pulse train, separated by $\lambda$, are shown - the experiment uses four. 


\section{The AWA Drive Beam}

Using the existing multibunch capabilities of the AWA, we have designed and constructed an experiment to demonstrate the multimode concept. The AWA can provide a train of electron bunches spaced at multiples of $760 \mathrm{ps,} \mathrm{with} \mathrm{energy} 15$ $\mathrm{MeV}$, emittance $20 \mathrm{~mm} \mathrm{mrad}$, and bunch length of 3-5mm. For this experiment we passed 4 electron bunches, separated by our minimum achievable distance of 760 ps, though a dielectric-lined tube.

\section{The Dielectric Waveguide}

There are four parameters that must be chosen for the waveguide: (see Fig. 1) $a, b$, $\varepsilon$ and $L$. The choice of inner radius $a$ is constrained, primarily, by the emittance and energy of the AWA drive beam. Due to the relatively large emittance and low energy, the inner radius of the tube must be kept large to maximize the charge transported through the tube. The downside of a large inner radius is that a relatively low gradient is excited, which makes it difficult to obtain a large total energy gain (loss) unless the tube is long. An inner radius of $0.5 \mathrm{~cm}$ and tube length of $60 \mathrm{~cm}$ was found to be capable of transmitting about $5 \mathrm{nC}$ which yields energy changes that are large enough to measure with a spectrometer. The choice of dielectric constant was based on finite group velocity effects and availability of materials. In order for the $r f$ packets of the 4 bunches to interact, the energy of the first bunch must not have propagated away by the time the fourth bunch enters the tube, therefore we want to keep the group velocity $\left(v_{g}\right)$ low. Since $v_{g} \sim c / \varepsilon$, we want $\varepsilon$ high. We chose $\varepsilon=38.1$ which gives $v_{g} \sim 0.026 c$. The dielectric material [5] is $\mathrm{CaTiO}_{3}-\mathrm{LaAlO}_{3}$ with a perovscit structure. With $a$ and $\varepsilon$ fixed, the outer radius $b$ was adjusted until the net wavelength of the wakefield in the multimode tube was equal to the distance between bunches. For the multiple beam experiment at AWA, the drive bunch spacing is $23.05 \mathrm{~cm}$ since $\lambda_{r f}$ is $23.05 \mathrm{~cm}$ at 1.3 $\mathrm{GHz}$; thus the outer radius $b$ is solved to be $1.44 \mathrm{~cm}$.

\section{EXPERIMENTAL SETUP}

The AWA drive linac was configured to provide a collinear bunch train of 4 bunches, each with charge $Q=5 \mathrm{nC}$, bunch length $\sigma_{Z}=4.5 \mathrm{~mm}$ and energy spread $(\triangle E / E)_{F W H M}=0.3 \%$. Each bunch must be spaced so that the individual wakes left by each bunch adds constructively to the wake of the preceding bunch. For the multimode experiment, simulation shows that the spacing between bunches must be accurate to within $\sigma_{Z}$ out of $\lambda$ (or $4.5 \mathrm{~mm}$ out of $230.5 \mathrm{~mm}$ ) for appreciable constructive interference, and hence large wakefields, to obtain.

\section{Optical Splitter}

A train of 4 laser pulses is made by optically splitting a single laser pulse into 4 separate pulses as shown in Fig. 2. The distance between laser pulses is adjusted optically by moving mirrors on translation stages in the delay line. Initially, the 
distance between bunches is crudely measured with a streak camera, using the $10 \mathrm{~ns}$ sweep rate, giving timing resolution of $\sim 10$ ps between bunches. Final bunch spacing must be done during the experiment by maximizing the deceleration of trailing bunches after they emerge from the multimode structure.

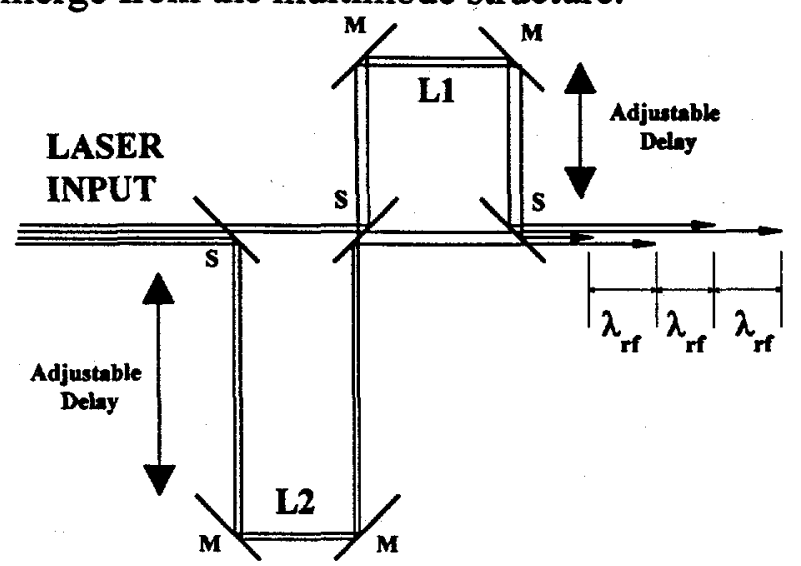

FIGURE 2. Laser Beam Splitter Optics. Three 50/50 beam splitters (S) in combination with four mirrors (M) are used to produce four laser pulses of variable separation. For this experiment, legs L1 and L2 are adjusted until the four pulses are separated by one wavelength of the $\mathrm{rf}\left(\lambda_{\mathrm{rf}}\right)$ driving the photoinjector. (Lines separated for clarity.)

\section{Spectrometer}

A central part of the experimental effort is to study the wakefield enhancement due to the bunch train; thus measurement of the energy spectrum of each drive beam is crucial. Also, because the 4 drive beams must be spaced to within one $\sigma_{Z}$ to achieve significant acceleration, a high demand is placed on the AWA's energy measurement system. The energy measurement system must have a resolution of $0.5 \%$ or better and be insensitive to position jitter since bunches within the train may not exactly follow the same path. To this end, a new imaging spectrometer was designed and built (Fig. 3 ) to provide improved momentum resolution over a wider range while reducing the sensitivity to beam jitter. Setting the tungsten slit to $300 \mu \mathrm{m}$, the resolution of the spectrometer is calculated to be $0.2 \%$. 


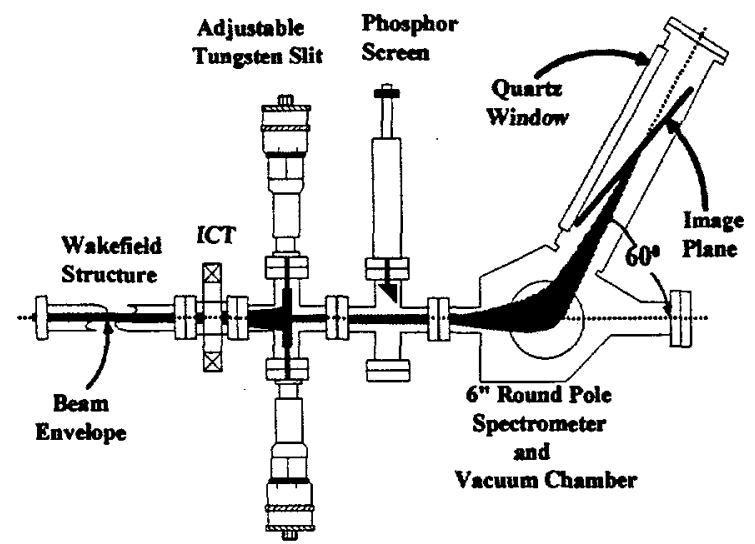

FIGURE 3. Energy Measurement System. The decelerated drive bunch train passes through a tungsten slit of adjustable width, which is imaged through a $60^{\circ}$ dipole (diameter $=6^{\prime \prime}$ ) onto a phosphor screen located at the image plane. The phosphor screen is viewed with an intensified camera through the quartz window.

\section{EXPERIMENTAL RESULTS}

In this section we first calculate the expected energy spectrum of the bunch train based on the beam parameters used during the run with our analytic model. We then compare this expected energy spectrum to the experimental data.

Using (3), the computed wakefield as a function of $\mathrm{z}$ (the distance behind the first bunch) for our specific experimental parameters is plotted in Fig. 4. The parameters used to create the plot were a charge of $4.8 \mathrm{nC}$, a rms. bunch length of $4.5 \mathrm{~mm}$, a FWHM energy spread of $0.3 \%$ and an initial energy of $15.46 \mathrm{MeV}$. From Fig. 4, one can see that peak accelerating wakefield is only about $1.2 \mathrm{MeV} / \mathrm{m}$.

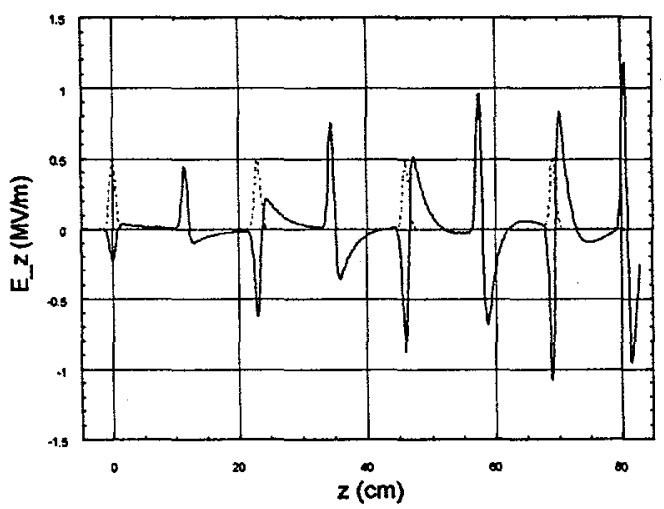

FIGURE 4. The wake excited (solid line) by 4 monochromatic drive beams (charge $=4.8 \mathrm{nC} / \mathrm{each}$ and bunch length $=4.5 \mathrm{~mm}$ ) spaced one wavelength $(23.05 \mathrm{~cm})$ apart. The beam distribution is shown as the dashed line and is moving right to left.

Using the computed wakefields (Fig. 4) one may easily extract the monochromatic energy spectrum generated by the 4 drive beams. Projecting the wake experienced by each particle within the bunch onto the energy axis and scaling by the appropriate 
value of $\mathrm{L}$ gives the monochromatic energy spectrum. The total energy spectrum is then computed by taking the convolution of the monochromatic energy spectrum over the energy distribution of the drive bunch, which is measured to be approximately Gaussian in shape.

The energy spectrum of the decelerated bunch train was measured at the image plane (Fig. 3) and acquired with a frame grabber for off-line analysis. For tube length $\mathrm{L}=59.87 \mathrm{~cm}$ and the parameters given earlier in this section, we plot the measured energy spectrum (Fig. 5, solid line) and compare it to the computed energy spectrum (Fig. 5, dotted line). For the purposes of this experiment, the figure of merit is the location of the energy peaks in Fig. 5. The intensity of the individual energy peaks (here, intensity is proportional to the charge) is meaningless for a simple reason. Namely, the transverse position of the individual bunches striking the $300 \mu \mathrm{m}$ wide tungsten slit of Fig. 3 fluctuate causing the amount of charge transported to the image plane to fluctuate. The energy peaks (in $\mathrm{MeV}$ ) from the data are located at 15.35 , $15.12,14.98$ and 14.88 while the fits peaks are located at $15.35,15.12,14.98$ and 14.86. As is readily seen from this data, the agreement between theory and experiment is excellent.

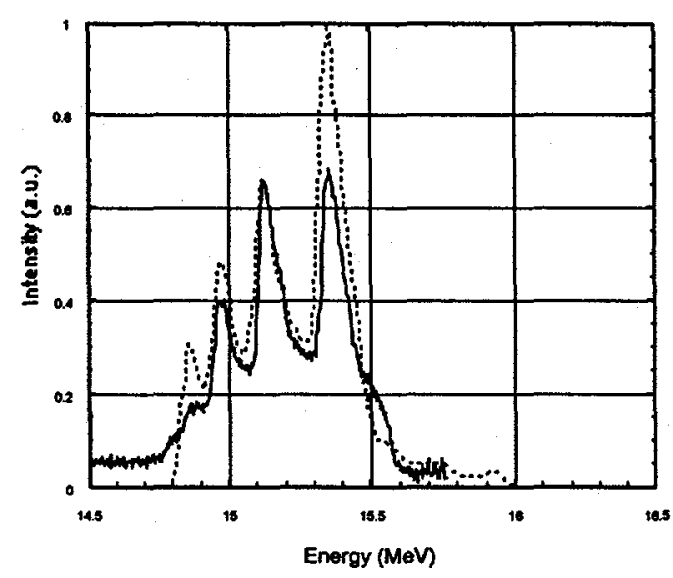

FIGURE 5. Energy spectrum of the $4 \times 4.8 \mathrm{nC}$ bunch train. The solid line is the measured energy spectrum and the dotted line is the analytic model.

To ensure that the measured energy spectrum was indeed due to multimode wakefields, we sent a low charge pulse train $(<1 \mathrm{nC}$ each) through the multimode structure. Since the wakefield excitation amplitude is proportional to the total charge we expect the total width of the energy spectrum to be narrower in this case than in the high charge case. The projected energy spectrum of the low charge case is shown in Fig. 6 . The narrow spread of the energy spectrum in Fig. 6 confirms that we are indeed seeing the effects of the multimode wakefield deceleration in the data of Fig. 4. 


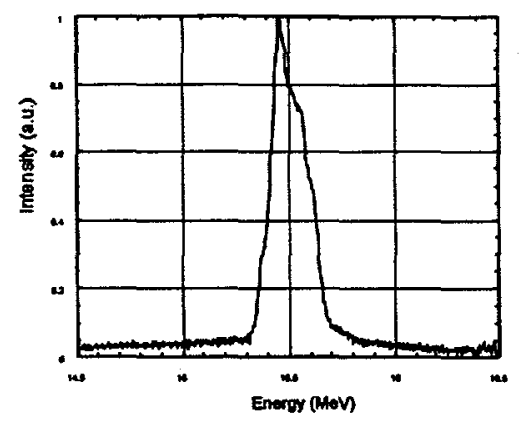

FIGURE 6. Energy Spectrum of low charge pulse train $(4 \mathrm{x} \sim 0.5 \mathrm{nC})$ with an average energy of $E_{0}=$ $15.46 \mathrm{MeV}$. Total energy spread is observed to be much less than in the high charge case.

\section{CONCLUSION}

We have experimentally demonstrated the concept of multibunch excited wakefields in a multimode dielectric structure. We found that the wakefield is indeed reinforced by a pulse train and the measurement is in complete agreement with the existing theory [3] from the conventional single mode DWFA based on linear superposition. Due to the fact that we used a dielectric tube with a relatively large inner radius, the observed gradient is low $(1.2 \mathrm{MV} / \mathrm{m})$. However, if we use the upgraded AWA gun (under construction) with $\mathrm{Q}=50 \mathrm{nC}$ and $\sigma_{\mathrm{Z}}=1 \mathrm{~mm}$, and modify the dielectric structure to have inner radius of $2 \mathrm{~mm}$ and outer radius of $11.5 \mathrm{~mm}$, a pulse train of 4 bunches can produce $145 \mathrm{MV} / \mathrm{m}$ accelerating gradient.

\section{ACKNOWLEDGEMENTS}

We appreciate the interesting ideas put forth by Dr. T-B. Zhang, Prof. J. L. Hirshfield, and Prof. T. C. Marshall and the useful discussions with Prof. J. Rosenzweig and Dr. J. Simpson. This work was supported by the US Department of Energy, Division of High Energy Physics, under contract W-31-109-ENG-38.

\section{REFERENCES}

[1] T-B. Zhang, J. L. Hirshfield, T. C. Marshall, B. Hafizi, Phys. Rev. E56 4647 (1997)

[2] J.G. Power, W. Gai, P. Schoessow, Phys. Rev. E60 6061 (1999)

[3] see M. Rosing, W. Gai, Phys. Rev, D42 1829 (1990) or K. Ng, Phys. Rev. D42 1819 (1990)

[4] P. Schoessow, M. E. Conde, W. Gai, R. Konecny, J. Power, J. Simpson, J. Appl. Phys 84663 (1998)

[5] St. Petersburg Electrical Engineering University, Physics Dept. 